\title{
Application of Page Ranking Algorithm Based on Numbers of Link Visits in Web Recommendation System for Online Business
}

\author{
${ }^{1}$ Mahesh Kumar Singh, ${ }^{2}$ Om Prakash Rishi, \\ and ${ }^{3}$ Sumit Wadhwa \\ ${ }^{1}$ Research Scholar, Department of Computer Science and Informatics, \\ University of Kota, Rajasthan, India \\ ${ }^{2}$ Director Research, University of Kota, Rajasthan, India \\ ${ }^{3}$ Assistant Professor, Department of Information technology \& Management, \\ Greater Noida, U.P., India \\ maheshkrsg@gmail.com,omprakashrishi@yahoo.com, sumitwadhwa1988@gmail.com
}

\begin{abstract}
World Wide Web plays a vital role in global information service center. Online business is growing very rapidly by creating websites. Website is made of number of webpages. Webpage is dynamic collection of hyperlink and usage information, providing rich sources of web data for web mining. Due to exponential growth of dynamic information over the internet, information overload create big challenges for the researchers in this area. One of the key components which ensure the acceptance of web page search service is the web page ranker a component which is said to have been the main contributing factor of Google. This paper discusses the page ranking algorithms based on contents, structures and usages in web mining, proposed page ranking algorithm based on number of links visit. Since every product has its own unique web page for its decryption hence in selection of top most visited links can be applicable to create the recommendation list for the corresponding customer in online business.
\end{abstract}

Keywords- PageRank, Web Mining, Recommendation System, Information retrieval, Web Graph, Online business.

\section{INTRODUCTION}

Web Mining is defined as the application of data mining techniques on the World Wide Web to find hidden information, This hidden information i.e. knowledge could be contained in content of web pages or in link structure of WWW [8] or in web server logs. WWW is a vast resource of hyperlinked and heterogeneous information including text, image, audio, video, and metadata. With the rapid growth of information sources available on the WWW and growing needs of users, it is becoming difficult to manage the information on the web and satisfy the user needs. Actually, we are drowning in data but starving for knowledge. Therefore, it has become increasingly necessary for users to use some information retrieval techniques to find, extract, filter and order the desired information.

A. Search engine [2] receives users query, processes it, and searches into its index for relevant documents i.e. the documents that are likely related to query and supposed to be interesting then, search engine 
ranks the documents found relevant and it shows them as results. This process can be divided in the following tasks:

B. Crawler [11,12] is in charge of visiting as many pages and retrieves the information needed from them. The idea is that this information is stored for the use by the search engine afterwards.

C. Indexing the information provided by a crawler has to be stored in order to be accessed by the search engine. As the user will be in front of his computer waiting for the answer of the search engine, time response becomes an important issue. That is why this information is indexed in order to decrease the time needed to look into it.

D. Searching The web search engine represents the user interface needed to permit the user to query the information. It is the connection between the user and the information repository.

E. Sorting/Ranking Due to the huge amount of information existing in the web, when a user sends a query about a general topic (e.g. java course), there exist an incredible number of pages related to this query but only a small part of such amount of information will be really interesting for the user. That is why the search engines incorporate ranking algorithms in order to sort the results.

F. Recommendation System There is individual page for every product and services provided by online business. Page ranking algorithm rank every page which gives the popularity of the web page $[9,10]$ that can be used to filter the most popular product. Today is the era of online businesses. There are billions of small and large companies that provide the online facilities of sale and purchase the product and services. Every product has the different links of its description. Hence PageRank is help to identify the popularity of the product.

This paper is categorized into seven parts. First part discusses the idea about the web mining and its types. Second part is the related study of page ranking algorithms; third part discusses the problems identification, forth part is implementation of page ranking algorithm based on number of link visits, fifth part discusses its application in online business, six part is comparison analysis, and last part discusses the conclusion of the paper.

\section{WEB MINING}

Extraction of interesting (non-trivial, implicit, previously unknown and potentially useful) information or patterns from large databases is called Data Mining. Web Mining is the application of data mining techniques to discover and retrieve useful information and patterns (knowledge) from the WWW documents and services web mining can be divided into three categories [1] as shown in figure 1.

a. Web Content Mining,

b. Web Structure Mining, \&

c. Web Usage Mining

A. Web Content Mining (WCM) describes the automatic search of information resources available online, and involves mining web data content. It is emphasis on the content of the web page not its links. It can be applied on web pages itself or on the result pages obtained from a search engine. WCM is differentiated from two different points of view: Information Retrieval (IR) View and Database View. In IR view, most of the researches use bag of words, which is based on the statistics about single words in isolation, to represent unstructured text. For the semi-structured data, all the works utilize the HTML structures insides the documents. For database view, Web mining always tries to infer the structure of the Web site to transform a Web site to become a database. 
B. Web Structure Mining (WSM) is used to generate structural summary about the Web sites and Web pages. The structure of a typical Web graph consists of Web pages as nodes and hyperlinks as edges connecting two related pages. Technically, WCM mainly focuses on the structure of inner-document, while WSM tries to discover the link structure of the hyperlinks at the inter-document level. Web structure mining tries to discover the model underlying the link structures of the Web. The model is based on the topology of the hyperlink with or without the link description. This model can be used to categorize the Web pages and is useful to generate information such as similarity and relationships between Web sites. And the link structure of the Web contains important implied information, and can help in filtering or ranking Web pages. In particular, a link from page A to page B can be considered a recommendation of page $B$ by the author of A. Some new algorithms have been proposed that exploit this link structure not only for keyword searching, but other tasks like automatically building a Yahoolike hierarchy or identifying communities on the Web. The qualitative performance of these algorithms is generally better than the IR algorithms since they make use of more information than just the contents of the pages. While it is indeed possible to influence the link structure of the Web locally, it is quite hard to do so at a global level. So link analysis algorithms that work at a global level possess relatively robust defenses against spamming.

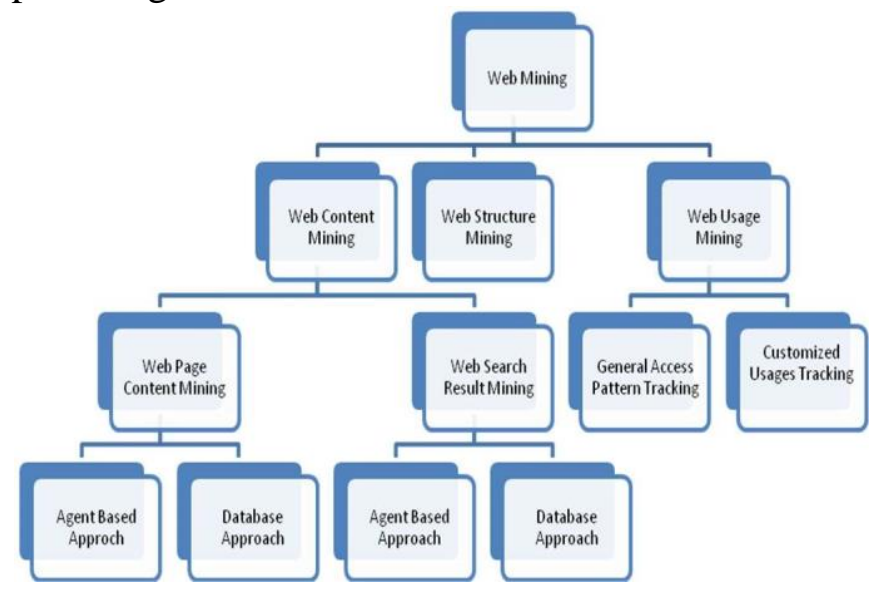

Fig. 1 Taxonomy of Web Mining

C. Web Usage Mining (WUM) tries to discover user navigation patterns from web data and the useful information from the secondary data derived from the interactions of the users while surfing on the Web. It focuses on the techniques that could predict user behavior while the user interacts with Web. This type of web mining allows for the collection of Web access information for Web pages. This usage data provides the paths leading to accessed Web pages. This information is often gathered automatically into access logs via the Web server. CGI scripts offer other useful information such as referrer logs, user subscription information and survey logs. This category is important to the overall use of data mining for companies and their internet/ intranet based applications and information access. The three categories of web mining described above have its own application areas including site improvement, business intelligence, Web personalization, site modification, usage characterization and page ranking etc. The search engines to find more important pages generally use the page ranking. Implement PRNLV method use web structure and web uses mining technique to rank web pages and its application in web recommendation system. 


\section{RELATED WORK OF RANKING ALGORITHAMS}

The web is very large and diverse and many pages could be related to a given query. That is why a method/algorithm is used to sort the entire pages subject to be interesting to a user's query. All the algorithms consider the web pages as a directed graph in which pages are denoted as nodes and links are denoted as edges.

\section{A. PageRank Algorithm (PR)}

Surgey Brin and Larry Page developed a ranking algorithm used by Google, named PageRank [2] after Larry Page (cofounder of Google search engine), that uses the link structure of the web to determine the importance of web pages [7]. It takes back links into account and propagates the ranking through links. Thus, a page has a high rank if the sum of the ranks of its back links is high. A simplified version of page rank is defined as follows

$$
P R(p)=(1-c) \sum_{q \in I(p)} \frac{P R(q)}{O(q)}
$$

In the calculation of PageRank a factor $\mathrm{c}$ is used for normalization. Note that $0<\mathrm{c}<1$ because there are pages without incoming links and their weight is lost. Later PageRank was modified observing that not all users follow the direct links on WWW.

$$
P R(p)=(1-d)+d \sum_{q \in I(p)} \frac{P R(q)}{O(q)}
$$

Where $\mathrm{d}$ is a dampening factor that is usually set to 0.85 (any value between 0 and 1), $\mathrm{d}$ can be thought of as the probability of users' following the links and could regard $(1-d)$ as the page rank distribution from non-directly linked pages .Consider the following directed graph [4]

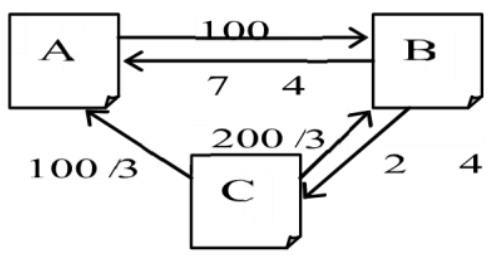

Fig. 2. Example Graph

The PageRanks for pages A, B, C in figure 2 are calculated by using (3.2) with $\mathrm{d}=0.5$, the page ranks of pages $\mathrm{A}, \mathrm{B}$ and $\mathrm{C}$ becomes: $\mathrm{PR}(\mathrm{A})=1.2, \mathrm{PR}(\mathrm{B})=1.2, \mathrm{PR}(\mathrm{C})=0.8$

\section{B. Weighted Page Rank Algorithm (WPR)}

Wenpu Xing and Ali Ghorbani [5] proposed an extension to standard PageRank called Weighted PageRank (WPR). It rank pages according to their importance not only consider link structure of web graph. This algorithm assigns larger rank values to more important pages instead of dividing the rank value of a page evenly among its outgoing linked pages. Each outlink page gets a value proportional to its popularity. The popularity is measured by its number of inlinks and outlinks.[6] 


$$
P R(p)=(1-d)+d \sum_{q \in I(P)} P R(q) W_{(q, p)}^{\text {in }} W_{(q, p)}^{\text {out }}
$$

Where Win (q,p) and Wout (q,p), for inlinks and outlins is given as

$$
\begin{gathered}
W_{(\boldsymbol{q}, p)}^{\text {in }}=\frac{I_{p}}{\Sigma_{v \in R(q)} I_{v}} \\
W_{(\boldsymbol{q}, \boldsymbol{p})}^{\text {aut }}=\frac{o_{p}}{\Sigma_{v \in R(q)} O_{v}}
\end{gathered}
$$

Where Iv,Ip and Ov, Op represent the number of inlinks and outlinks of page $\mathrm{v}$ and page p respectively. The Page Ranks for pages A, B, C are calculated by using (3.3) with $\mathrm{d}=0.5$, the page ranks of pages $\mathrm{A}$,

$\mathrm{B}$ and $\mathrm{C}$ are $\mathrm{PR}(\mathrm{A})=0.65, \mathrm{PR}(\mathrm{B})=0.93, \mathrm{PR}(\mathrm{C})=0.60$.

\section{Page Content Rank Algorithm (PCR)}

Jaroslav Pokorny and Jozef Smizansky [3] gave a new ranking method of page relevance ranking employing WCM technique, called Page Content Rank (PCR). This method combines a number of heuristics that seem to be important for analyzing the content of web pages. The page importance is determined on the basis of the importance of terms, which the page contains. The importance of a term is specified with respect to a given query q. PCR uses_a neural network as its inner classification structure. The importance of a page $\mathrm{P}$ in PCR is calculated as an aggregate value of the importance of all terms that $\mathrm{P}$ contains. For a promotion of the significant term and a suppression of the others, the second moment is again used as an aggregate function [2] Page_importance $(\mathrm{P})=\sec \_$moment $(\{\operatorname{importance}(\mathrm{t}): \mathrm{t} P\})(3.4)$

\section{Hyperlinked Induced Topic Search Algorithm (HITS) [13, 24]}

This algorithm assumes that for every query topic, there is a set of "authoritative" or "authority" pages/sites that are relevant and popular focusing on the topic and there are "hub" pages/sites that contain useful links to relevant sites including links to many related authorities as shown in figure 3.

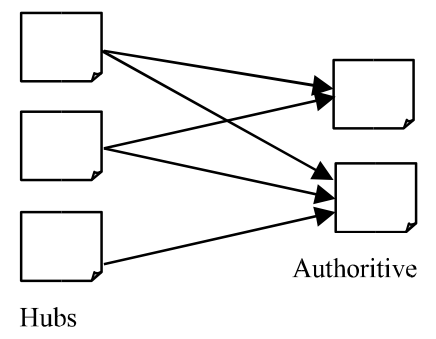

Fig 3. Hubs and Authorities

\section{E. Working of HITS}

The HITS works in two phases Sampling and Iterative in the Sampling phase a set of relevant pages for the given query are collected i.e. a sub-graph $S$ of $G$ is retrieved which is high in authority pages. The Iterative phase finds hubs and authorities using the output of the sampling phase using following equations. 


$$
\begin{aligned}
& H_{p}=\sum_{q \in I(p)} A_{q} \\
& A_{p}=\sum_{q \in B(p)} H_{q}
\end{aligned}
$$

Where Hp is the hub weight, Ap is the Authority weight, I (p) and B(p) denotes the set of reference and referrer pages of page $\mathrm{p}$.

\section{PROBLEMS \& ISSUES OF THE PAGE RANKING ALGORITHAMS}

The main problems and issues of discussed page ranking algorithms are summarized as:

A. Rank quality of PageRank: The discussed ranking algorithms have shown a really high quality and the proof is that success of Google (or they are still being used) successfully. However, some improvements can be done on it.

B. Data Mining Technique of PageRank: PageRank algorithm used only Web Structure Mining and Web Content Mining technique; it does not use Web Usage Mining, which may significantly improve the quality of rank of web pages according to users information needs.

C. PageRank is Static in Nature: In PageRank algorithm, the importance or rank score of each page are static in nature. The rank changes only with link structure of web.

\section{PAGE RANKING ALGORITHAMS BASED ON NUMBERS OF LINK VISITS (PRNLV)}

PRNLV (Page Ranking based on Link Visits) based on Web Structure Mining and Usage Mining; it takes the user visits of pages/links into account with the aim to determine the importance and relevance score of the web pages. To accomplish the complete task from gathering the usage characterization till final rank determination many subtasks are performed such as

A. Storage of user's access information (hits) on an outgoing link of a page in related server log files.

B. Fetching of pages and their access information by the targeted web crawler.

C. For each page link, computation of weights based on the probabilities of their being visited by the users.

D. Final rank computation of pages based on the weights of their incoming links.

E. Retrieval of ranked pages corresponding to user queries.

\section{A. Calculation of Visits (hits) of links}

If $\mathrm{p}$ is a page with outgoing-link set $\mathrm{O}(\mathrm{p})$ and each outgoing link is associated with a numerical integer indicating visitcount ( $\mathrm{VC}$ ), then the weight of each outgoing link connecting to page $\mathrm{p}$ to page o is calculated by

$$
\text { Weight }_{\text {link }}(p, q)=\frac{\overline{V C}(p, q)}{\sum_{q \in O(p)} V C\left(p, q^{\prime}\right)}
$$

B Page Rank based on Numbers Link Visit (PRNLV)

If $\mathrm{p}$ is a page having inbound-linked pages in set $\mathrm{B}(\mathrm{p})$, then the rank (PRNLV) is given equation (5.2)

$P R L K(p)=(1-d)+d\left(\sum_{b \in B(p)} P R L L(b)\right.$ Weight $\left.(b, p)\right)$ 
where $\mathrm{d}$ is the damping factor as is used in PageRank, Weightlink is the weight of the link calculated by (5.1).The iteration method is used for the calculation of page rank. Example fig 2 Taking $d=0.5$, these equations can easily be solved using iteration method the final results obtained are:

$\operatorname{PRNLV}(\mathrm{A})=1.08, \operatorname{PRNLV}(\mathrm{B})=1.26, \operatorname{PRNLV}(\mathrm{C})=0.66$

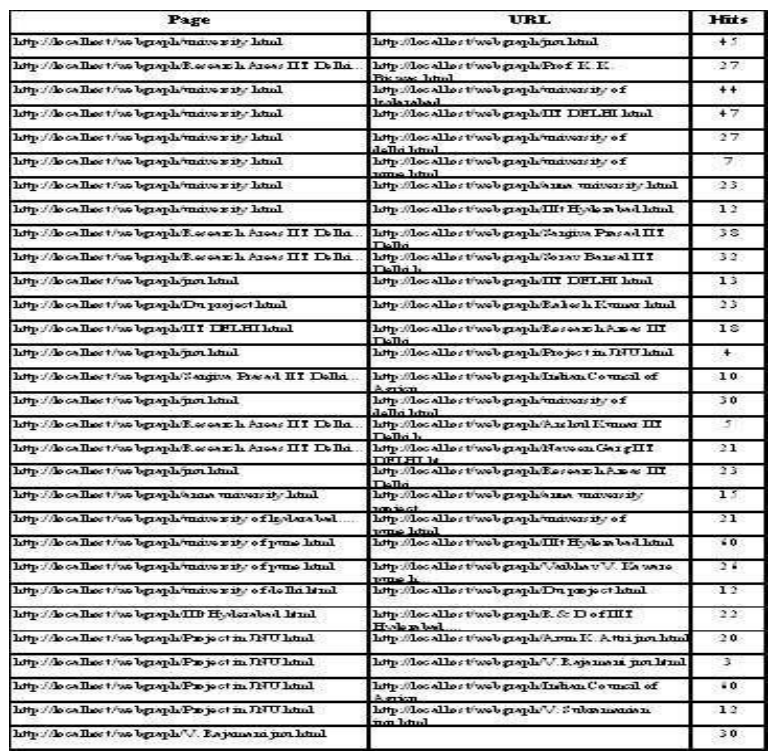

Fig. 4. Numbers of the Hits of the links

\section{Experimental Evaluation}

To evaluate the performance and effectiveness of the proposed algorithm a prototype is designed a website is created using HTML,CSS and Java script, wamp server is used as the server to store the activities of the users. The web graph of the web site as shown in figure 4. The data are collected by installing the model on the local host and uses are able to log the web site using their email as the ID, visit to the different links. Every page has different URL with their visit count as shown in figure 4.Hence compute the page rank of each link (page) using the formula (5.2) and arrange these links in its decreasing order of their page rank as shown in figure 5. Hence the highest ranked page on the top of the list. The variation of page rank of the pages in the web site shown in the figure 6 .

\begin{tabular}{|c|c|c|}
\hline Eeb Rage & PR & FRIIT \\
\hline ann a univers ity projet.html & 0.2929 & 0.12 \\
\hline anna university.html & 0.1686 & 0.452 \\
\hline Anshul Kumar IIT Delhi.html & 0.2499 & 0.87 \\
\hline Arun Kumar Attri jnu .html & 0.1876 & 0.287 \\
\hline Du Projecthtml & 0.675 & 0.321 \\
\hline IIIT Hyder ab ad.html & 0.135 & 0.34 \\
\hline IITD elhi.html & 0.4053 & 0.2344 \\
\hline Indian Council of Agricultural Research.html & 0.1698 & 0.76 \\
\hline jun.html & 0.123 & 0.1453 \\
\hline KG Saxena JNU.html & 0.564 & 0.15 \\
\hline $\begin{array}{l}\text { N aveen } G \text { arg IIT DELHI.html } \\
\text { Prof. KK Bisinass,html }\end{array}$ & $\begin{array}{l}0.187 \\
0.345\end{array}$ & $\begin{array}{l}0.198 \\
0.2879\end{array}$ \\
\hline $\begin{array}{l}\text { Prof. KK Bisiwas.html } \\
\text { Project in JNU.htmI }\end{array}$ & $\begin{array}{l}0.345 \\
0.463\end{array}$ & $\begin{array}{l}0.2679 \\
0.209\end{array}$ \\
\hline R \& D of IIIT Hyderabad.htmI & 0.122 & 0.673 \\
\hline R ankesh Kumar.html & 0.2897 & 0.2897 \\
\hline Research Areas IIT D elhi.html & 0.4542 & 0.4298 \\
\hline Sanjiuga Pras ad IIT Delhi.html & 0.242 & 0.546 \\
\hline Saurabh Bansal IIT Delhi.html & 0.1674 & 0.1897 \\
\hline U nivesity of Delhi.html & 0.134 & 0.456 \\
\hline Univesity of Hyder ab ad.htmI & 0.1276 & 0.1988 \\
\hline U nivesity of Pune.html & 0.1342 & 0.564 \\
\hline U nivesity.html & 0.286 & 0.78 \\
\hline V.Rajamani jnu.html & 0.242 & 0.342 \\
\hline V.Subramani ami jnu.html & 0.675 & 0.765 \\
\hline Vaibhav $\vee$ Kaware pune.html & 0.1765 & 0.76 \\
\hline
\end{tabular}

Fig. 5. Page Rank Using (PR and PRNLV)

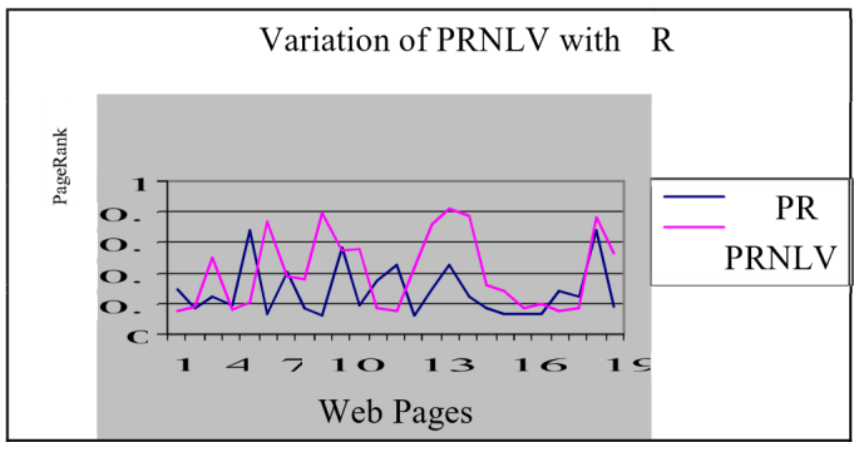

Fig. 6. Variation of PRNLV with PR 


\section{APPLICATION OF PAGE RANKING IN RECOMMENDATION SYSTEM}

Every on line commercial company uses the specified pages for specified product that contains the specification of the product on their web site. Every page has its own unique URL hence the popularity of the product can be computed by the popularity of the links that is page rank of the page. Recommendation System tries to identify the user's interest in the specific domain of contents based on their previous experiences. When a user interact with the E-commercial site helshe offers a set of implicit or explicit information like clicks, rating, comments etc. about his/her taste. Association Rule mining play a vital to associate the e-mail ID with the page with its rank that can be helpful in creating the recommended list for the specified customer.

\section{COMPARISION OF PRNLV WITH PR \& WPR}

From the table 1 it is clear that PRNLV is much better than PR and WPR.

Table 1: Comparison of Page ranking Algorithms

\begin{tabular}{|l|l|l|l|}
\hline $\begin{array}{l}\text { Algorithm } \\
\text { Parameter }\end{array}$ & PageRank (PR) & $\begin{array}{l}\text { Weighted Page Rank } \\
\text { (WPR) }\end{array}$ & $\begin{array}{l}\text { Page Rank based on Numbers } \\
\text { Link-Visit (PRNLV) }\end{array}$ \\
\hline Description & $\begin{array}{l}\text { Computes scores at indexing time. } \\
\text { Results are sorted according to } \\
\text { importance of pages. }\end{array}$ & $\begin{array}{l}\text { Computes scores at indexing } \\
\text { time. Results are sorted } \\
\text { according to importance of } \\
\text { pages. }\end{array}$ & $\begin{array}{l}\text { Computes scores at indexing } \\
\text { time. Pages are sorted } \\
\text { according to importance and } \\
\text { relevance. }\end{array}$ \\
\hline $\begin{array}{l}\text { Mining } \\
\text { Technique } \\
\text { Used }\end{array}$ & $\begin{array}{l}\text { Web } \\
\text { Structure Mining }\end{array}$ & $\begin{array}{l}\text { Web } \\
\text { Structure Mining }\end{array}$ & $\begin{array}{l}\text { Web Structure } \\
\text { Mining, Web Usage } \\
\text { Mining }\end{array}$ \\
\hline Rank & Ranks are & Ranks are & Ranks are unequally \\
\hline Distribution & $\begin{array}{l}\text { equally distributed to outgoing } \\
\text { links. }\end{array}$ & $\begin{array}{l}\text { equally distributed to outgoing } \\
\text { links. }\end{array}$ & $\begin{array}{l}\text { distributed among outgoing } \\
\text { links according to } \\
\text { probabilities of visit. }\end{array}$ \\
\hline $\begin{array}{l}\text { I/P } \\
\text { Parameters }\end{array}$ & Inbound links of pages & $\begin{array}{l}\text { Inbound links and Outbound } \\
\text { links of pages }\end{array}$ & $\begin{array}{l}\text { Inbound links, } \\
\text { Outbound links, Visit } \\
\text { Counts of links. }\end{array}$ \\
\hline $\begin{array}{l}\text { Working } \\
\text { levels }\end{array}$ & n* & n* & No \\
\hline Complexity & O(log n) \\
\hline $\begin{array}{l}\text { Nature of } \\
\text { Rank }\end{array}$ & $\begin{array}{l}\text { Less dynamic (rank changes with } \\
\text { link structure })\end{array}$ & $\begin{array}{l}\text { Less dynamic (rank changes } \\
\text { with link structure })\end{array}$ & $\begin{array}{l}\text { More dynamic (rank changes } \\
\text { with visit counts \& structure } \\
\text { of links) }\end{array}$ \\
\hline Relevancy of pages & No & O(log n) \\
\hline
\end{tabular}


Gyancity Journal of Electronics and Computer Science Vol.3, No.2, pp. 1-10, September 2018 ISSN:2446-2918 DOI: 10.21058/gjecs.2018.32001

\begin{tabular}{|l|l|l|l|}
\hline Importance of pages & Yes & Yes & Yes \\
\hline Quality of result & Low & High & High \\
\hline Advantages & $\begin{array}{l}\text { Computation of } \\
\text { ranks with minimum } \\
\text { effort and less complexity. }\end{array}$ & $\begin{array}{l}\text { Computation of ranks with } \\
\text { effort and less complexity. }\end{array}$ & $\begin{array}{l}\text { Pages returned are of high } \\
\text { quality and relevancy as user } \\
\text { feedbacks are taken into } \\
\text { account. Search space can be } \\
\text { very much pruned as pages } \\
\text { are sorted according to users' } \\
\text { information needs. }\end{array}$ \\
\hline Limitations & $\begin{array}{l}\text { No relevancy of pages is } \\
\text { considered in rank computation. } \\
\text { All links are considered equally } \\
\text { important. }\end{array}$ & $\begin{array}{l}\text { No relevancy of pages is } \\
\text { considered in rank computation. } \\
\text { All links are considered equally } \\
\text { important. }\end{array}$ & $\begin{array}{l}\text { Extra effort on crawlers to } \\
\text { fetch the visit counts of pages } \\
\text { from web servers. Extra } \\
\text { calculations to find the } \\
\text { weights of links. }\end{array}$ \\
\hline
\end{tabular}

\section{CONCLUSION}

Mining of knowledgeable data from a huge amount of data is very complex task, World Wide Web information play a vital role for information collection and sharing. The ranking algorithms are used to search the relevance information in very efficient manner. Different page ranking algorithms are used in different techniques. The PRNLV uses the user's browsing information in consideration to calculate rank of a documents rather than link structure. Due to browsing information in consideration PRNLV system is more dynamic than other ranking algorithms. The popularity of the web pages (products) computed by the page ranking algorithm can be used for performance of the products in the on line business.

\section{REFERENCES}

[1] N. Duhan, A. K. Sharma, K. K. Bhatia, "Page Ranking Algorithms: A Survey", 2009 IEEE International Advance Computing Conference (IACC 2009) Patiala, India, 6-7 March 2009.

[2] A Broder. A taxonomy of web search. Technical report, IBM Research, 2002.

[3] Jaroslav Pokorny, Jozef Smizansky, Page Content Rank: An Approach to the Web Content Mining.

[4] A. Broder, R. Kumar, F. Maghoul, P. Raghavan, R. Stata. Graph structure in the web. In In Proceedings of the 9th International World Wide Web Conference, 2000.

[5] W Xing,A. Ghorbani, "Weighted PageRank Algorithm", Proceedings of the Second Annual Conference on Communication Networks and Services Research (CNSR`04), 2004 IEEE

[6] G. Salton . C. Buckley. "Term Weighting Approaches in Automatic Text Retrieval. In Information Processing and Management. Vol. 24, No. 5, pp. 513- 5231998.

[7] S. Chakrabarti, B. E. Dom, S. R. Kumar, P. Raghavan, S. Rajagopalan, A. Tomkins, D. Gibson, and J. Kleinberg, Mining the Web's link structure. Computer, 32(8):60-67, 1999.

[8] T. B.-Lee, R. Cailliau, A. Luotonen, H.F. Nielsen, A. Secret. "The World-Wide Web. Communications of the ACM", 37(8):76-82, 1994.

[9] M.G Campana, F. Delmastro:” Recommernder Systems for online and mobile Social Networks: A survey”, Online Social Networks and Media3-4(2017), pp 75-97 
[10] Q. Su , L. Chem:" A Method for discovering cluster of e-Commerce in terest patterns using Click- stream data". Electronic

Commerce Research and Applications. 1567-4223 Elsevier-2014 http://dx.doi.org/10.1016/j.elerap.2014.10.002

[11] B. Pinkerton." Finding what people want: Experiences with the web crawler". In The second Internation WWW Conference Chicago, 1994.

[12] J. Cho, H. Garc'ýa-Molina, L. Page. "Efficient crawling through URL ordering". Computer Networks and ISDN Systems, 30(1-7):161-172, 1998.

[13] C. Ding, X. He, P. Husbands, H. Zha, H. Simon. "Link analysis: Hubs and authorities on the world", Technical report:47847, 2001.

[14] A. Borodin, G. O. Roberts, Jeffrey S. Rosenthal, P. Tsaparas. Finding authorities and hubs from link structures on the world wide web. In World Wide Web, pages 415-429, 2001.

[15] S. L. Yong, M. Hagenbuchner Ah Chung Tsoi, "Ranking Web Pages using Machine Learning Approaches", IEEE/WIC/ACM International Conference on Web Intelligence and Intelligent Agent Technology, 2008.

\section{Author's Profile}

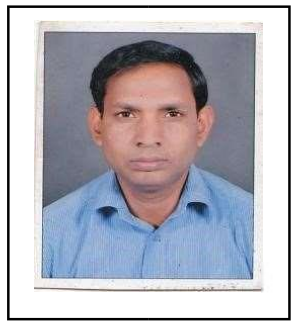

Mahesh Kumar Singh received his B.Tech from UPTU Lucknow in 2004 , M.Tech. from Jamia Hamdard University, New Delhi in 2012 and Perusing Ph D in Computer Science, Kota University on web mining and its application to web business intelligence.

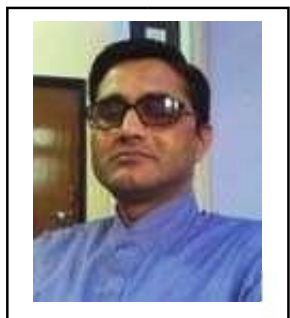

Dr. Om Prakash Rishi is Director of Research University of Kota and Head of Department of Computer Science \& Informatics. His Research areas are Artificial Intelligence, Intelligent Systems \& CBR, Software Systems and Agent technology, Cloud Computing, Information Security.

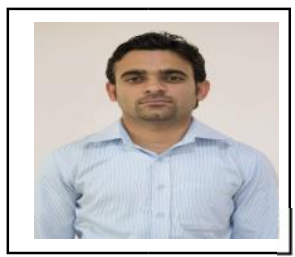

Sumit Wadhwa received his B.Tech from Kurukshetra University, Kurukshetra in 2009 , M.Tech. from Kurukshetra University, Kurukshetra, in 2013 and research areas are Wireless Sensor Network \& Artifical intelligence. 